\title{
BUENAS PRÁCTICAS EN EMPRENDIMIENTOS LÁCTEOS, DESDE LA ECONOMÍA SOCIAL Y SOLIDARIA EN BIBLIÁN-ECUADOR
}

\author{
Ángel Esteban Muñoz Alvarez* \\ https://orcid.org/0000-0002-9172-9694 \\ Jorge Edwin Ormaza Andrade** \\ https://orcid.org/0000-0001-5449-1042 \\ Yonimiler Castillo Ortega*** \\ https://orcid.org/0000-0002-7710-5199
}

\section{RECIBIDO: Agosto 2021 / ACEPTADO: Octubre 2021 / PUBLICADO: Enero 2022}

Como citar: Muñoz Alvarez, Angel; Ormaza Andrade, Jorge; Castillo Ortega, Yonimiler. (2022). Buenas prácticas en emprendimientos lácteos, desde la economía social y solidaria en Biblián-Ecuador. Telos: revista de Estudios Interdisciplinarios en Ciencias Sociales, 24 (1), Venezuela. (Pp. 40-61).

DOI: www.doi.org/10.36390/telos241.04

\section{RESUMEN}

La actividad láctea ha demostrado con su cadena valor y cantidad de empleos que genera su relevancia en la economía ecuatoriana ante las adversidades socioeconómicas que ha experimentado en los últimos tiempos, desde el enfoque social y solidario, para reactivar el desarrollo productivo en el país. En este contexto, el presente artículo tuvo por objetivo general explorar las buenas prácticas de la producción láctea, desde la economía social y solidaria en el cantón Biblián-Ecuador. Basado en una investigación descriptiva y transversal se recolectó la información en tres empresas asociativas de producción de leche ubicadas en el mencionado cantón, siendo los agentes informantes 55 socios de las referidas asociaciones, quienes respondieron a un cuestionario estructurado. A la luz de los resultados, con la tabulación y análisis de los datos se hace una propuesta de buenas prácticas, con sus respectivas estrategias. Se implementó la herramienta CANVAS para generar un modelo de negocio que impulse las estrategias y subsane las debilidades del proceso lácteo, procurando la mejora continua y el desarrollo de los productores de leche de Biblián-Ecuador, al tiempo que promueven el desarrollo social y solidario en la poblacional cantonal y del país.

\footnotetext{
* Maestrante de la Maestría en Desarrollo Local mención Economía Social y Solidaria Universidad Católica de Cuenca. Correo electrónico: angel.muñoz.24@est.ucacue.edu.ec

** Docente de la Universidad Católica de Cuenca Sede Azogues, Carrera de Administración de Empresas - Candidato a Doctor en el programa Doctoral en Ciencias Sociales mención Gerencia de la Universidad del Zulia - Venezuela. Cuenca, Ecuador. Correo electrónico: jormaza@ucacue.edu.ec

*** Docente de la Universidad Católica de Cuenca, Jefatura de Posgrados - Carrera de Economía. Cuenca, Ecuador. Correo electrónico: ycastillo@ucacue.edu.ec
} 
Palabras clave: Buenas prácticas de emprendimiento, actividad láctea, economía social y solidaria, desarrollo social, productores de leche.

\section{Good practices in dairy enterprises, from the social and solidarity economy in Ecuador}

\section{ABSTRACT}

The dairy activity has demonstrated with its value chain and the number of jobs it generates its relevance in the Ecuadorian economy in the face of the socioeconomic adversities it has experienced in recent times, from a social and solidarity approach, to reactivate productive development in the country. In this context, the general objective of this article was to explore the good practices of dairy production, from the social and solidarity economy in the canton of BibliánEcuador. Based on a descriptive and cross-sectional research, information was collected in three associative enterprises of milk production located in the mentioned canton, being the informant agents 55 partners of the referred associations, who responded to a structured questionnaire. In light of the results, with the tabulation and analysis of the data, a proposal of good practices is made, with their respective strategies. The CANVAS tool was implemented to generate a business model that promotes the strategies and remedies the weaknesses of the dairy process, seeking continuous improvement and development of the milk producers of Biblián-Ecuador, while promoting social development and solidarity in the cantonal population and the country.

Key words: Good entrepreneurial practices, dairy activity, social and solidarity economy, social development, milk producers.

\section{Introducción}

La economía social y solidaria (ESS), inicia en el continente europeo en el siglo XIX, en ese tiempo no se alcanzaba la armonía social, de esa forma se programó la asociación de la clase obrera como una alternativa para el desarrollo y se logró mejorar las condiciones de vida de las personas basándose en los principios de solidaridad (Da Ros, 2001), en procura de mejorar las condiciones de vida de la población afectados por fenómenos como la globalización (Ojeda, 2009), y con el tiempo se ha convertido para Razeto (1999), en una propuesta de transformación social en el campo científico.

La economía social y solidaria ha rechazado las indiferencias individualistas que propician el utilitarismo y ha hecho cuestionamientos sobre las formas de producción y maneras de comprender las realidades de los paradigmas económicos dominantes en la contemporaneidad (Gibson y Graham, 2011). Con su evolución, se ha nutrido de otros enfoques y perspectivas, y de acuerdo con la Red Intercontinental de Promoción de la Economía Social y Solidaria (2015, p. 1), es "una alternativa al capitalismo y a sistemas económicos autoritarios controlados por el estado"

Para Fernández y Falconí (2012) y Sarria Icaza (2002), esta teoría se ha configurado de tal manera que más allá de ver las prácticas de producción, comercialización y desarrollo integral del ser humano en comunidad, se fundamenta en la cooperación, solidaridad, democracia, igualdad y sostenibilidad del ser humano en los procesos económicos, otorgando importancia a la producción cooperativa, comercialización justa, así como al consumo responsable y finanzas 
Buenas prácticas en emprendimientos lácteos, desde la economía social y solidaria en BibliánEcuador

éticas, que para Coraggio, en entrevista de Subijana y Russo (2011), se conjuga todo en el Buen Vivir, pues tiene su basamento en el bien común.

Para Mogrovejo, Mora y Vanhuynegem (2012), los primeros pasos que se dieron en relación con esta corriente en América Latina ocurrieron en Argentina, Brasil, México y Venezuela en el siglo XIX, y fue a partir de las primeras décadas del siglo XX que se desarrolló en el resto de los países de la región, a través de emprendimientos cooperativos, que de acuerdo a Bretos et al. (2018) se centran en la confianza y la cooperación.

En el Ecuador, Zhunio (2021, p. 15), señala que "a partir de la constitución de Montecristi 2008 la economía social y solidaria obtiene el reconocimiento formal, e introduce en el sistema legislativo que la economía en la nación (...)". Así, en la constitución nacional (Asamblea Constituyente, 2008, p. 136), artículo 283 establece que: "El sistema económico es social y solidario (...) y tiene por objetivo garantizar la producción y reproducción de las condiciones materiales e inmateriales que posibiliten el buen vivir", donde el ser humano es el centro de cualquier actividad económica en la nación.

En ese contexto nacional de desarrollo social, la industria láctea tiene una participación importante, pues de acuerdo Lácteos Latam (2020, párr. 1), es una actividad económica dinámica dentro de la industria manufacturera, "más de un millón de personas trabajan en la cadena productiva de leche, siendo un importante músculo en la reactivación económica del país (...) y representa el $14 \%$ del PIB Agroalimentario". Asimismo, el Centro de la Industria Láctea del Ecuador (S/F, p.1) señala que:

"La importancia económica y social de la Cadena Láctea es cada vez mayor, pues al ser una de las más dinámicas dentro de la industria manufacturera, contribuye con alrededor del $1 \%$ del total del Producto Interno Bruto (PIB), y representa el $5,4 \%$ del Producto Interno Bruto Industrial (BCE).

Esta actividad tiene gran influencia en la economía nacional, aunado a que más del $60 \%$ de los productores lo conforman pequeños y medianos productores campesinos, en donde participan en gran medida los grupos familiares y generan empleo en las zonas rurales, especialmente, ello coadyuva social y económicamente al desarrollo local y de acuerdo a Tello (2006), es una dinámica socio-económica en un área específica, resultante del comportamiento, acciones e interacciones de los productores como agentes socio-económicos, que se esfuerzan para mantener la sostenibilidad de la calidad de la vida de los habitantes del cantón que se dedican a esa actividad, usando eficazmente los recursos que poseen.

Dichos esfuerzos han sido redoblados, pues de acuerdo con el Centro de la Industria Láctea del Ecuador (2020), el COVID-19, generó alarmas en los productores ante alteraciones del comportamiento del consumidor, cambios tanto en los hábitos de consumo de leche, como en las preferencias y cantidad de demanda de los productos nacionales, lo cual condujo a los productores a innovar en sus gestiones y motivó a que se planteara como objetivo general de este trabajo explorar las buenas prácticas de la producción láctea, desde la economía social y solidaria en el cantón Biblián-Ecuador. En tal sentido, en el siguiente apartado se presentan ampliamente a las empresas sociales y solidarias; seguidamente, se hizo un acercamiento a las buenas prácticas en emprendimientos lácteos; lo cual condujo a indagar en los encuestados su 
percepción al respecto. Los resultados obtenidos sentaron las bases para elaborar una propuesta para el emprendimiento lácteo, así como un modelo de negocio.

\section{Empresas de la economía social y solidaria: generalidades y buenas prácticas}

La empresa social y solidaria tomó cuerpo a voluntad de algunas asociaciones voluntarias para generar empleo a un grupo de personas que históricamente estaban fuera del contexto laboral tradicional, y de empresarios individuales que querían administrar negocios, pero con marcados objetivos sociales, pues, al distanciarse de la empresa tradicional, fueron ganando adeptos que observaban la inclusión como un medio generador de empleo, en un medio por demás competitivo (Fonteneau et al., 2010).

En este sentido, hay que diferenciar una empresa social y solidaria de una de corte tradicional o capitalista. En lo social, la propiedad no es necesariamente colectiva, puesto que su objetivo se orienta a una vinculación laboral en forma colectiva y solidaria más no enfocada en ganancias, esto en virtud del tipo de productos o servicios que comercializan, ya que su mercado se define en contextos de masificación de la producción para beneficios mayores, en cambio, las otras empresas son lucrativas y se enfocan en la ganancia (Rodríguez y Sánchez, 2016).

Señala Gómez Calvo (2013, p. 114), que la economía solidaria se sustenta en un modelo de desarrollo local como alternativa al modelo exógeno que impera en la actualidad, y en ese sentido agrega que este último modelo quita a individuos y colectivos la "...capacidad para tomar decisiones económicas, puesto que éstas son tomadas por agentes externos al área local, mientras que en los patrones de desarrollo endógeno las variables son controladas por los mismos agentes interesados".

Al respecto, Ruiz Revelo (2015), expresa que las empresas de ESS se identifican por un desarrollo dirigido hacía un interés común, puesto que son generadores de empleo, trabajan con habitantes de la comunidad formando un crecimiento empresarial que busca mayor productividad otorgando bienestar a los habitantes. De hecho, el surgimiento de la economía solidaria coincide con la creación de cooperativas de crédito, mutualista y asociaciones (Fajardo, 2009), instituciones que en palabras de Ruiz Revelo (2015), son actores económicos y sociales presentes en los sectores de la sociedad para atender las necesidades de la población.

En cuanto a la gestión de estas organizaciones, se identifica dos grandes dimensiones: la dimensión social, ética y cultural, y la dimensión económica. La primera, relacionada con la gestión innovadora, enfocada al talento humano, conocimiento, cambio, pero además colaborativa y de responsabilidad social. Lo cultural y ético está orientada a la sensibilidad social, fomentando los valores, cooperación, autogestión y reciprocidad, complementariedad, armonía, respeto y equidad (Orrego y Arboleda, 2006), entendidos estos componentes se persigue lograr el bienestar social y mejora en la calidad de vida. La segunda, se articula a los procesos básicos de gestión (planificación, organización, dirección y control) y de recursos, es decir, agrupa a las actividades de acuerdo con la responsabilidad, reconocidos como finanzas, mercadeo, producción, servicio e información.

En este sentido, Razeto (1999) apunta a una gestión sin dependencia del Estado para evitar la injerencia en las decisiones; Singer (2002), en la misma línea es proclive a una gestión colectiva, en la cual participan las personas que quieran unirse a otras comunitariamente para generar emprendimientos económicos; mientras que Coraggio, referido por Subijana y Russo 
Buenas prácticas en emprendimientos lácteos, desde la economía social y solidaria en BibliánEcuador

(2011), expresa que como acción colectiva puede contrarrestar las tendencias sociales negativas de un sistema para construir un sistema económico alternativo.

Desde las dimensiones social y económica planteadas por Orrego y Arboleda (2006), y dadas las características particulares de estas empresas de la ESS, es imprescindible la consideración de un marco de buenas prácticas que permita y facilite su funcionamiento, pero a la vez se corresponda con su génesis. Al respecto, la organización no gubernamental Economistas sin Fronteras (EsF) (2017), en el informe de evaluación y buenas prácticas del proyecto 'Yo hago prácticas en la economía social y solidaria', aboga por generar una experiencia guiada por principios, objetivos y procedimientos que respondan a una normativa 0 parámetro consensuado, con miras al alcance de resultados positivos, que muestren la eficacia y utilidad en un contexto establecido. Así, la Red de Redes de Economía Alternativa y Solidaria (2011), menciona como parámetros el comercio justo, finanzas, ética, relaciones sociales, medio ambiente y ecología.

Desde esta perspectiva, Ayala Zaldaña y Berltrán Flores (2011), identifican un conjunto de principios para una correcta implementación de la ESS: unidad en la diversidad; membrecía voluntaria; igualdad de todas las organizaciones; justicia, equidad y democracia al interior de la organización; desarrollo basado en la persona; toma de decisiones participativas; y, educación permanente. Así mismo, la Ley Orgánica de Economía Popular y Solidaria y del Sector Financiero Popular y Solidaria (Asamblea Nacional de la República del Ecuador, 2011), establece en su art. 4, que las personas y organizaciones deben regirse por los siguientes principios: búsqueda del Buen Vivir y del bien común; primacía del trabajo sobre el capital; equidad de género; respeto a la identidad cultural; autogestión; responsabilidad social y ambiental; comercio justo; solidaridad y rendición de cuentas; $y$, distribución equitativa y solidaria de excedentes.

En esa misma tónica, Guerra (2012) y Fajardo (2018), estipulan que entre las características principales de una empresa social y solidaria se encuentran: democracia, las decisiones se toman de manera participativa; equidad, distribución equitativa de los derechos y obligaciones de los participantes; justicia distributiva, se procura una remuneración y distribución de las ganancias lo más justa posible; cuidado del medio ambiente, objetivos ecológicos en el funcionamiento de la empresa; y, producción de satisfactores, bienes o servicios que se requieran para satisfacer necesidades. Considerando estos planteamientos, a continuación se pone en contexto las buenas prácticas en emprendimientos lácteos, específicamente pertenecientes a la economía social y solidaria.

\section{Buenas prácticas en emprendimientos lácteos de la economía social y solidaria}

Para la creación de un organismo, institución o empresa, se requiere de motivación y sobre todo de personas emprendedoras, líderes optimistas que deseen cumplir metas y sobre todo mejorar la calidad de vida. Chong-González (2017) y Pérez Paredes et al., (2020), coinciden en que una persona emprendedora facilita no sólo procesos de innovación social y empresarial, sino promueve cambios para la generación de proyectos y métodos alternativos de producción de bienes y/o servicios.

En ese sentido, el emprendimiento está directamente relacionado con el crecimiento económico y la definición de políticas de innovación social y formación de empresa solidarias, a 
este se puede, por una parte, entender como una estrategia para producir el desarrollo comunitario y mitigar problemas sociales, por otra se considera un modelo para construir procesos de aprendizaje en las comunidades (González Ibarra, 2020). Hay que hacer anotaciones sobre el emprendimiento que tiene varias interpretaciones y una de ellas, es la que refiere a la construcción de proyectos para la creación de empresas bajo un enfoque solidario, sostenible y productivo para impulsar el desarrollo comunitario (Castelao, 2016).

En el Ecuador, los emprendimientos y prácticas en la Economía Popular y Solidaria (EPS), son de vital importancia, puesto que según el Instituto Nacional de Economía Popular y Solidaria (2014), el 64\% del empleo por subsector económico corresponde a la EPS, lo cual cambia la matriz productiva del país. Lo anterior, es ratificado por la Superintendencia de la Economía Popular y Solidaria (2021), al destacar que en el período que abarca desde el 2012 al 2021, el número de organizaciones ha crecido en un $177 \%$, y el número de personas vinculadas al sector ha crecido en un $281.3 \%$.

Como parte del emprendimiento en el Ecuador, en el sector de la economía social y solidaria, se encuentran las organizaciones dedicadas a la producción lechera. Se trata de un sector por demás fundamental y estratégico para el país ya que de acuerdo con el Centro de la Industria Láctea del Ecuador (2021, p. 1):

"...miles de personas viven de la leche y toda su cadena productiva: al menos 270 mil personas se dedican de manera directa a actividades de la industria, y más de 1,2 millones de personas en Ecuador dependen de la producción, transporte, transformación y comercialización de leche y productos lácteos".

En virtud de lo planteado, con miras a garantizar buenas prácticas para la adecuada producción en estos emprendimientos lácteos dentro de la ESS, y considerando lo establecido por la Organización de las Naciones Unidas para la Alimentación y la Agricultura (FAO) y la Federación Internacional de la Leche (FIL) (2012), es preciso que los productores consideren y apliquen las siguientes buenas prácticas agrícolas: la sanidad animal, higiene del ordeño, la nutrición (alimentos y agua), bienestar animal, el medio ambiente y gestión socioeconómica.

La sanidad animal, es clave para la productividad, en este sentido una buena práctica en la producción lechera consiste en establecer rebaños con resistencia a enfermedades, establecer una gestión sanitaria eficaz, utilizando productos químicos y medicamentos conforme a las prescripciones. En lo que respecta, a la higiene del ordeño, es importante garantizar que mientras se realice no se lesione a los animales, ni introduzcan contaminantes en la leche; es decir, que el ordeño se realice en condiciones higiénicas y la leche se manipule correctamente después del ordeño.

La nutrición (alimentos y agua), se refiere a garantizar un suministro adecuado y de buena calidad de alimentos y agua; el control de las condiciones de almacenamiento de los alimentos, y la trazabilidad de los alimentos adquiridos fuera de la explotación. En ese sentido, la calidad y salubridad de la leche que produce, así como la salud y productividad de un animal lechero, depende en gran medida de que se le proporcione los alimentos y el agua apropiados

Bienestar animal, como buena práctica, implica mantener a los animales libres de hambre, sed y malnutrición, así como de incomodidades, dolores, lesiones y enfermedades. Animales libres de temores para desarrollar un comportamiento animal normal. Un animal en malas condiciones salubres, con desnutrición no podrá producir acorde a lo esperado. Por su 
Buenas prácticas en emprendimientos lácteos, desde la economía social y solidaria en BibliánEcuador

parte, el medio ambiente, se refiere al impacto en la degradación de la tierra, la contaminación del aire, contaminación hídrica, una buena práctica en la explotación lechera que no tenga efectos adversos en el medio ambiente local, disponer de un sistema gestión de residuos adecuados. Finalmente, la Gestión socioeconómica, consisten en aplicar una gestión eficaz y responsable de los recursos humanos, garantizar que las actividades de la granja lechera se ejecuten de manera segura y competente y administrar la empresa para asegurar su viabilidad financiera.

Complementariamente, en el contexto ecuatoriano, Amagua Puga et al., (2016) identifican entre las buenas prácticas de las industrias lecheras, de manera general: la localización de la planta, el diseño y construcción, los servicios de planta (facilidades), los equipos y utensilios, monitoreo de los equipos, requisitos higiénicos (personal), materias primas e insumos, operaciones de producción, envasado, empaquetado y etiquetado, almacenamiento y aseguramiento de la calidad. El empaquetado y etiquetado como buena práctica se fundamenta en el Reglamento Sanitario de Etiquetado de Alimentos procesados para el consumo humano (Ministerio de Salud Pública, 2014).

Por su lado, el Reglamento de Buenas Prácticas para Alimentos Procesados (Presidencia de la República, 2002), indica que las buenas prácticas de manufactura son los principios básicos y prácticas generales de higiene en la manipulación, preparación, elaboración, envasado y almacenamiento de alimentos para consumo humano, con el objeto de garantizar que los alimentos se fabriquen en condiciones sanitarias adecuadas y se disminuyan los riesgos inherentes a la producción.

En suma, la ESS persigue el desarrollo local mejorando las condiciones de vida, y al existir varias asociaciones productoras de leche a nivel nacional se vuelve indispensable implementar las buenas prácticas en el procesamiento de la materia prima utilizada, y en todo lo que ello implique. En ese sentido, es conveniente revisar las buenas prácticas de estas asociaciones comunitarias en el Cantón Biblián.

\section{Abordaje Metodológico}

Se trata de una investigación descriptiva, con un diseño transversal, puesto que se realizó el levantamiento de datos en un espacio determinado, en este caso el Cantón Biblián, durante el mes de junio de 2021. La población de estudio estuvo constituida por las tres empresas asociativas de producción de leche adscrita y autorizada por el Departamento de Economía Popular y Solidaria del Gobierno autónomo descentralizado distrital del Cantón Biblián. Adicionalmente, estas organizaciones se encuentran regentadas por la Ley de Economía Popular y Solidaria. Siendo las unidades de observación, el total de socios que las componen, es decir 55 socios, mismos que se indican en el gráfico No. 1. 


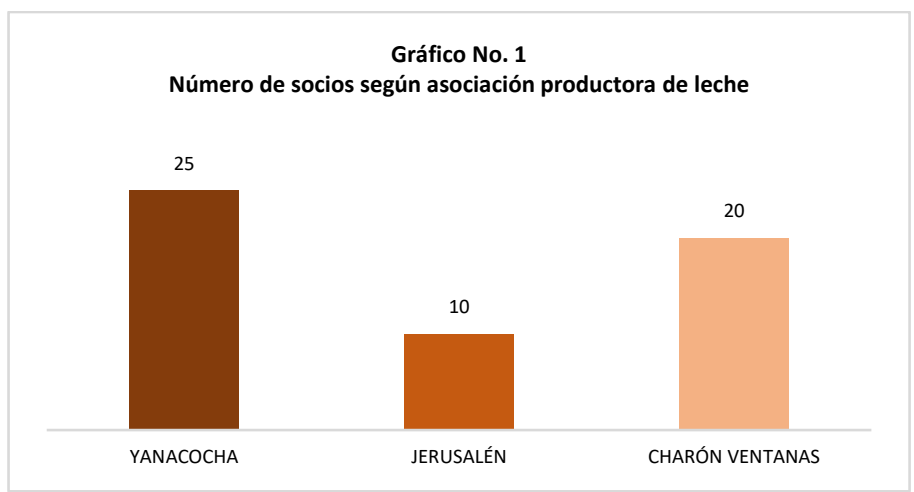

Fuente: Elaboración propia

Posteriormente, se levantó la información en la población de estudio, con el fin de diagnosticar los procesos de la producción lechera y la inclusión de las buenas prácticas, esto, mediante una encuesta compuesta por veinte preguntas de las cuales siete fueron dicotómicas y trece en escala Likert (en diversos formatos), cuyo instrumento fue el cuestionario previamente elaborado (de los cuales solo se presentan los más relevantes por espacio en este artículo, 7 items de escala y 6 dicotómicos, específicamente). La confiabilidad del instrumento se basa en la aplicación al número de socios de las organizaciones de producción de leche del cantón Biblián, así también, el instrumento es válido pues se enfoca en la medición de la aplicación de las buenas prácticas en dichas organizaciones. Esto, sirvió para obtener una visión de la realidad estudiada, sobre cómo se está realizando la producción y comercialización de la leche en mayor profundidad y veracidad, pues a través del contacto directo con los distintos actores implicados (productores), se obtuvo información certera acerca de la producción y del cumplimiento de buenas prácticas de economía social y solidaria.

Finalmente, se tabularon los datos, presentándose los mismos mediante gráficos estadísticos, donde se analizó en primera instancia, si las asociaciones tienen conocimiento sobre la economía social y solidaria, y buenas prácticas. A su vez, se indagó sobre el uso e importancia de las buenas prácticas. Es así que, con estos resultados se trazaron estrategias para la inclusión de las buenas prácticas de economía social y solidaria enfocadas a la producción lechera.

\section{Percepción de los encuestados}

La visión de los socios de las asociaciones lácteas, recogida a través del cuestionario realizado, se muestra a continuación: Con relación al conocimiento general sobre la Economía Social y Solidaria, un 70,91\% dice conocer que la organización forma parte del sector de la ESS, pero ello contrasta con un $63,64 \%$ que afirma desconocer el concepto, y, por tanto, las implicaciones de este modelo de organización. Ahora bien, cuando se pregunta sobre las buenas prácticas, un $60 \%$ señala conocer su significado, mientras que un porcentaje significativo (40\%) no sabe sobre las mismas (Gráfico No. 2). 
Buenas prácticas en emprendimientos lácteos, desde la economía social y solidaria en BibliánEcuador

Los resultados muestran la imperante necesidad de ofrecer formación a los miembros de las asociaciones, en particular, pero a la población en general acerca de las organizaciones de la ESS, su estructura y bondades, así como sobre las buenas prácticas, de tal manera que cada uno de ellos tengan claridad y conozcan a cabalidad la importancia de estos, para su aplicación en las entidades. Pero más allá de ello, pueda realmente constituir un sector que apunte al desarrollo socio-económico del país. En ese sentido, la generación de políticas públicas hacia el sector resulta necesaria.

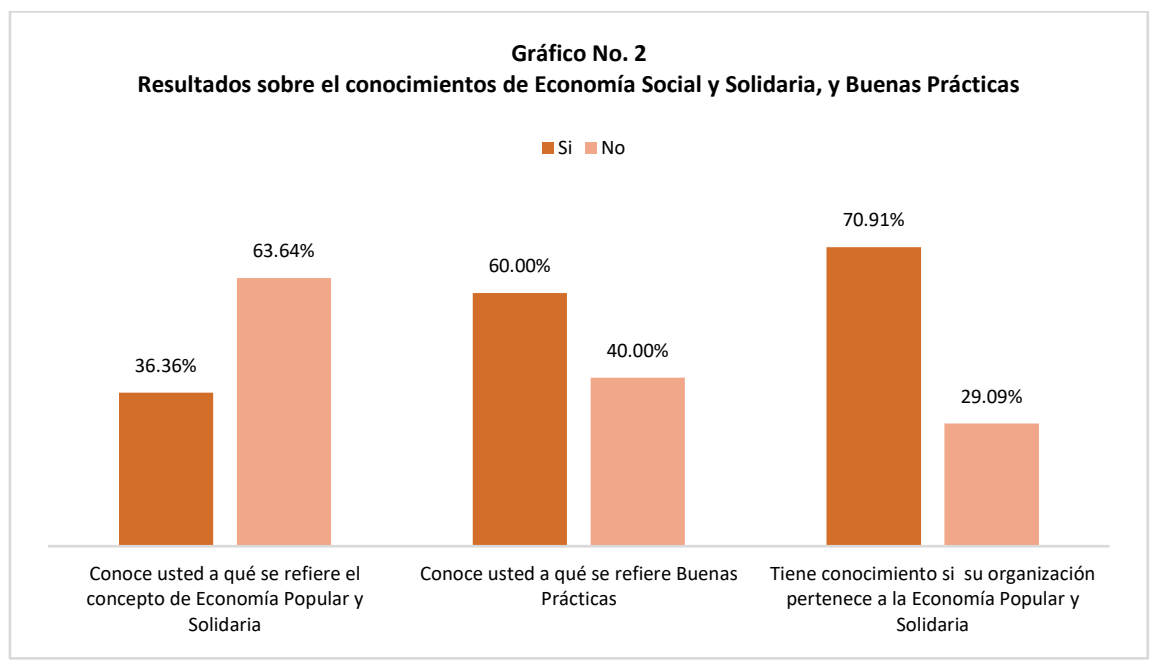

Fuente: Elaboración propia.

Cuando se preguntó, si los miembros de las asociaciones conocen del desarrollo de buenas prácticas de la economía popular y solidaria, en el gráfico 3 se muestra que el $61,83 \%$ de los socios indicaron que sí (suma de las tres organizaciones), mientras que $38,18 \%$ indican que tienen desconocimiento al respecto. Destaca, en el grado de desconocimiento la asociación productora de leche "Jerusalén" en donde existen graves falencias. Este resultado, deja claro que aun teniendo declaradas las buenas prácticas, resulta cuesta arriba aplicarlas, dado el porcentaje de desconocimiento existente. Sin embargo, al indagar acerca de las buenas prácticas conocidas, se identificaron las siguientes: capacitaciones, técnica de buen ordeño, manejo de registro, generar gastos de ingresos, crear fuentes de empleo en la comunidad y comercialización de lácteos. 


\section{Gráfico No. 3}

En la organización a la cuál usted pertenece conoce si se desarrollan Buenas Prácticas en relación a la Economía Popular y Solidaria

$61.82 \%$

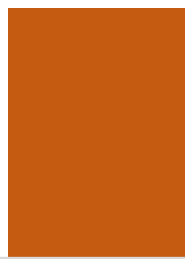

$\mathrm{SI}$
$38.18 \%$

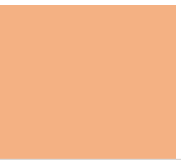

No

Fuente: Elaboración propia.

Al indagar sobre la existencia de una guía, manual o modelo de buenas prácticas que sea el instrumento base para el entendimiento de estos principios, enmarcados en la economía social y solidaria, el $64,29 \%$ señaló que no cuentan con algún instrumento, lo que muestra que, desde el punto de vista formal, dichas organizaciones adolecen de herramientas orientadas al apoyo de la gestión de las buenas prácticas, y, por ende, puede verse afectado el alcance de sus objetivos (ver gráfico No. 4).

Este resultado es incongruente con la presencia, en las asociaciones, de una dirección encabezada por un empresario "socialmente responsable", según lo afirma el $60,71 \%$ de los consultados. Pues un líder socialmente responsable, promueva el desarrollo de instrumentos dirigidos a garantizar el ejercicio de la responsabilidad social a través de las buenas prácticas.

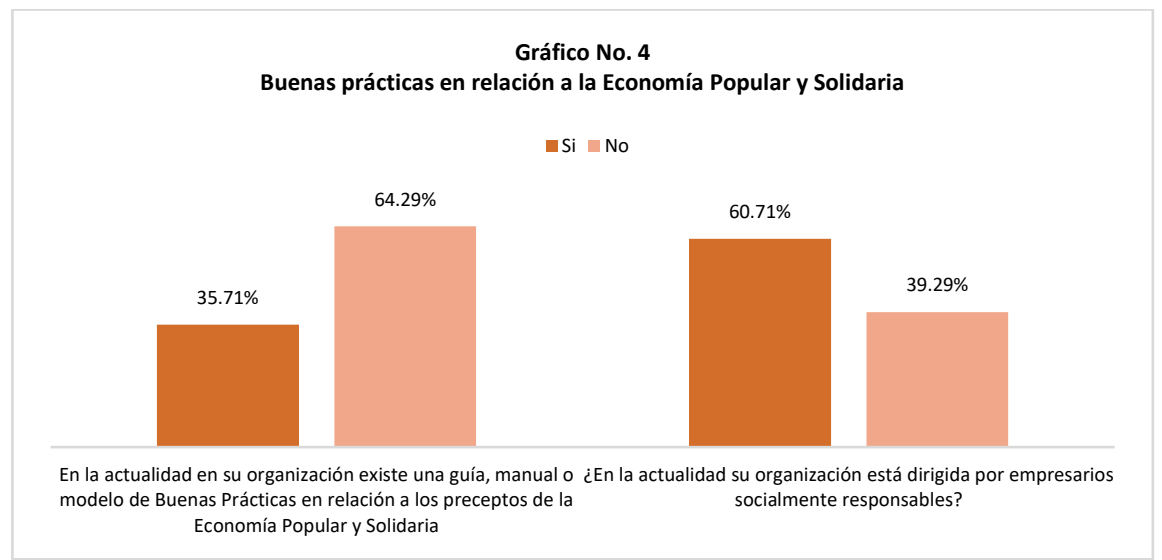

Fuente: Elaboración propia.

Ahondando el estudio hacia el uso de buenas prácticas en las asociaciones, se preguntó acerca de la existencia del control de calidad de los productos generados en las organizaciones 
Buenas prácticas en emprendimientos lácteos, desde la economía social y solidaria en BibliánEcuador

lácteas (Gráfico No. 5); en este caso el $58,18 \%$ aseguró que siempre existe un control de calidad de los productos, mientras que el $23,64 \%$ se dividido entre casi siempre, el $14,55 \%$ a veces y el $3,64 \%$ nunca. Estas respuestas en torno al control de calidad muestran que existen debilidades que deben ser atendidas en esta materia.

Por otra parte, al indagar si existe una higiene adecuada en la producción de leche y sus derivados (Gráfico No. 5), un porcentaje considerable (20\%) respondió que nunca, lo cual es indicativo de las fallas presentes en la producción e indudablemente repercuten en la calidad de los productos, y estaría asociado a las debilidades en el control de calidad.

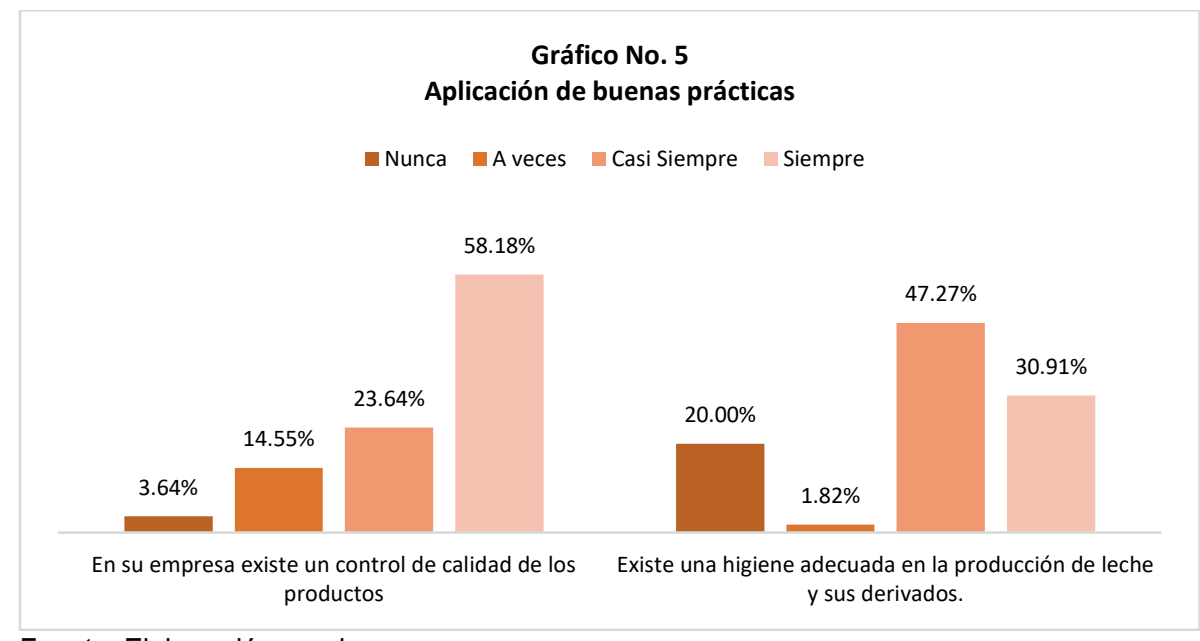

Fuente: Elaboración propia.

En cuanto a la importancia de la implementación de buenas prácticas dentro de una entidad para promover el desarrollo comunitario, las respuestas mostraron una tendencia entre muy importante $(47,27 \%)$ e importante $(40 \%)$. Al preguntar acerca de la concordancia entre las buenas prácticas y la mejora en la competitividad y compromiso del personal de las asociaciones lácteas, la tendencia estuvo entre muy importante $(52,73 \%)$ e importante $(45,45 \%)$ (Gráfico No. 6). En ambos casos, parece haber en los consultados, conciencia de la relevancia de las buenas prácticas tanto al interior de las asociaciones como al exterior de ellas. Es decir, al contar con procesos internos fortalecidos en las buenas prácticas, los resultados mostrados pueden conllevar a una mayor competitividad en el mercado, redundando es resultados positivos para el sector lácteo, y, por lo tanto, para la comunidad de socios de las organizaciones asociativas. 


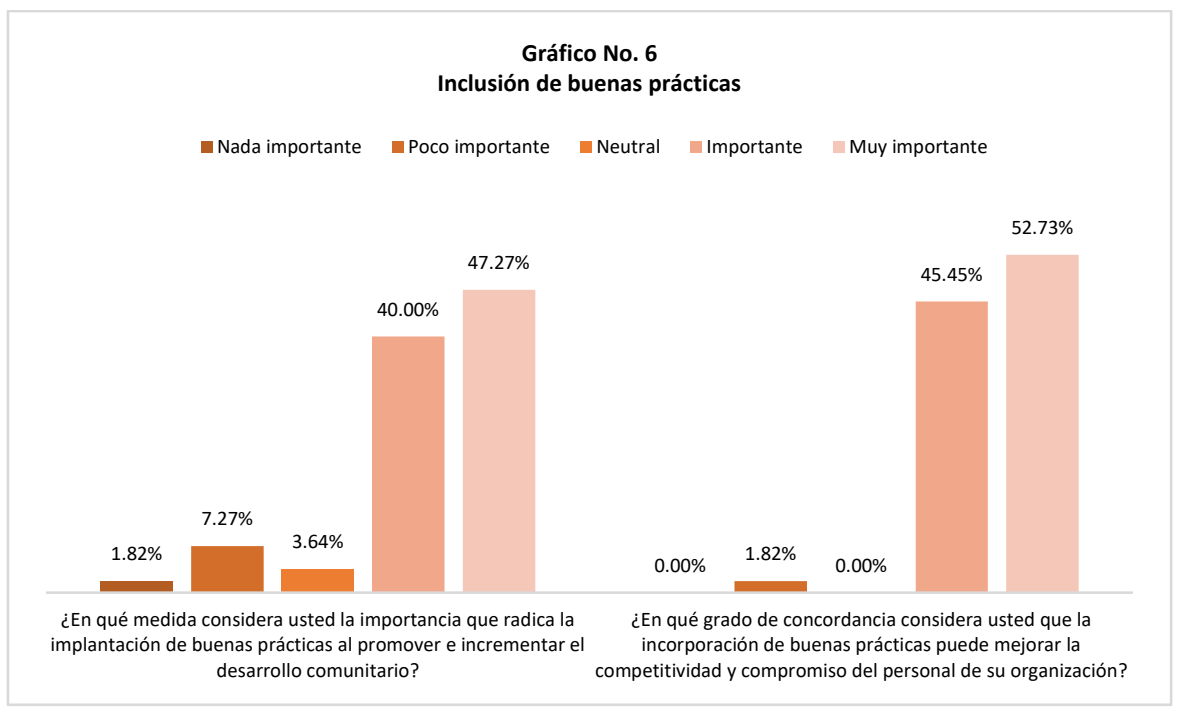

\section{Fuente: Elaboración propia.}

Finalmente, en el Gráfico No. 7, se observa que la población de estudio muestra la importancia de la implementación de buenas prácticas en la organización, debido a que estás incrementan la capacidad de gestión de los directivos $(69,09 \%$ y se garantiza la sostenibilidad y sustentabilidad en la producción de la leche y sus derivados $(45,45 \%)$. De igual manera, estuvieron totalmente de acuerdo $(45,45 \%)$ en que al implementar una guía de buenas prácticas se puede mejorar la imagen y posicionamiento de la asociación láctea.

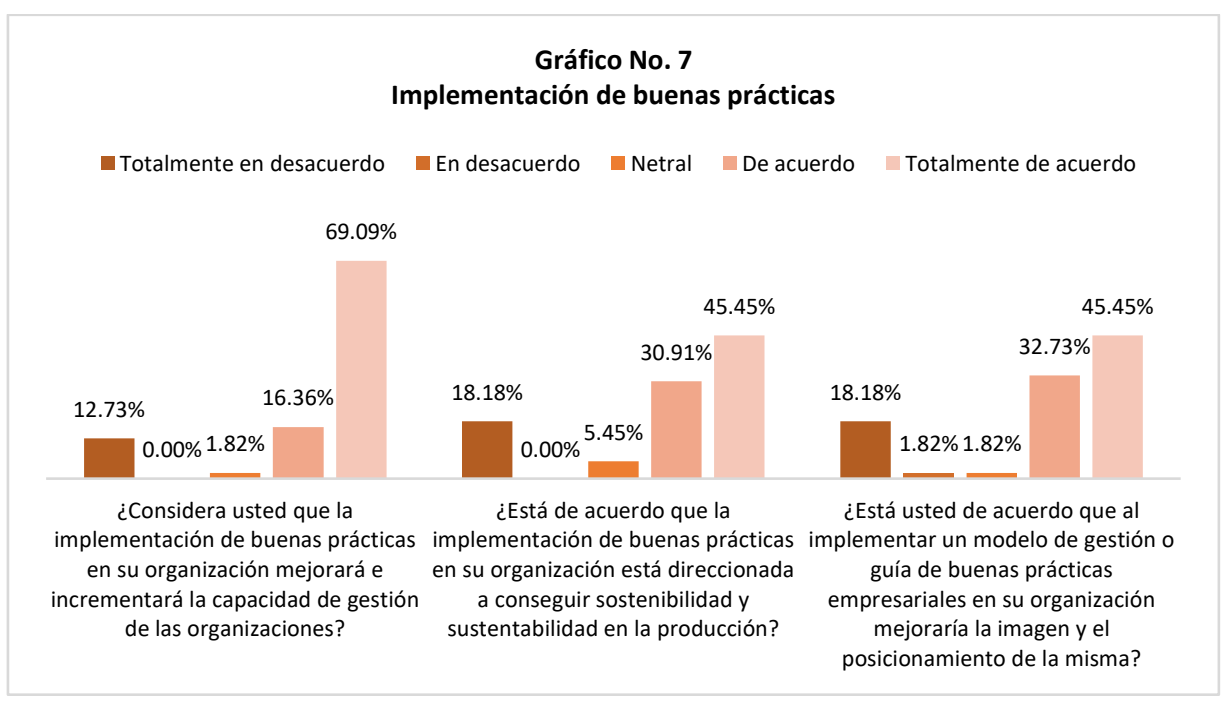


Buenas prácticas en emprendimientos lácteos, desde la economía social y solidaria en BibliánEcuador

En términos generales se puede aseverar que conocen del desarrollo de buenas prácticas de la economía popular y solidaria, en el gráfico 3 se muestra que el $61,83 \%$ de los socios indicaron que sí. Esos aspectos demarcan un direccionamiento claro sobre los esfuerzos organizacionales, y el trabajo mancomunado ético del personal, por la consecución de los objetivos, desde criterios de calidad, rentabilidad, competitividad, en conjunción con la sustentabilidad y el desarrollo comunitario, entre otros, en pro de prácticas empresariales integrales. De allí que se considere pertinente desarrollar en el siguiente apartado un modelo de negocios para las productoras de leche desde el marco social y solidario del Ecuador.

En el Ecuador se ha realizado investigaciones con respecto a las buenas prácticas en la producción lechera, entre estas la desarrollada por Bonifaz García y Requelme (2011), quienes determinan entre las buenas prácticas de ordeño a la higiene del ordeño, infraestructura, manejo de la leche y condición sanitaria de la leche, cuyos resultados reflejan que existe una ausencia de higiene en la rutina de ordeño, pues consideran que es una pérdida de tiempo, mal funcionamiento del equipo de ordeño, los desinfectantes y selladores no son utilizados de manera correcta, no identifican los agentes infecciosos, no la efectividad de las medidas de control y tratamientos, En relación a estas causas, la solución se basa en contar con los procedimientos claros, capacitación al personal y la existencia de un plan de seguimiento estricto de los procesos.

A nivel internacional, Villoch y Ponce (2010), generaron la guía de buenas prácticas de producción de leche para Cuba, relacionada con los indicadores de calidad entre estas se encuentran, la salud animal (mastitis), el manejo y bienestar animal (alimentación y agua), higiene del ordeño y post ordeño, registro, personal y protección del medio ambiente. Uno de los principales problemas detectados es que los productores no cuentan con infraestructura adecuada y no trabajan para obtener leche de calidad, por ello, la guía cuenta con 226 requisitos, clasificados en 4 grupos a ser implementadas de manera paulatina, es decir, aplicar y cumplir una fase a la vez para lograr el cumplimiento total de la misma de manera óptima.

\section{Propuesta de buenas prácticas y modelo de negocio para las empresas asociativas productoras de leche, desde la economía social y solidaria.}

A la luz de los resultados, con la tabulación y análisis de los datos se presenta de forma sucinta en la figura 1, la propuesta de buenas prácticas para las empresas asociaciones productoras de leche, con sus respectivas estrategias. Cada una de las cuales se amplían más adelante en la tabla 1. 
Figura 1

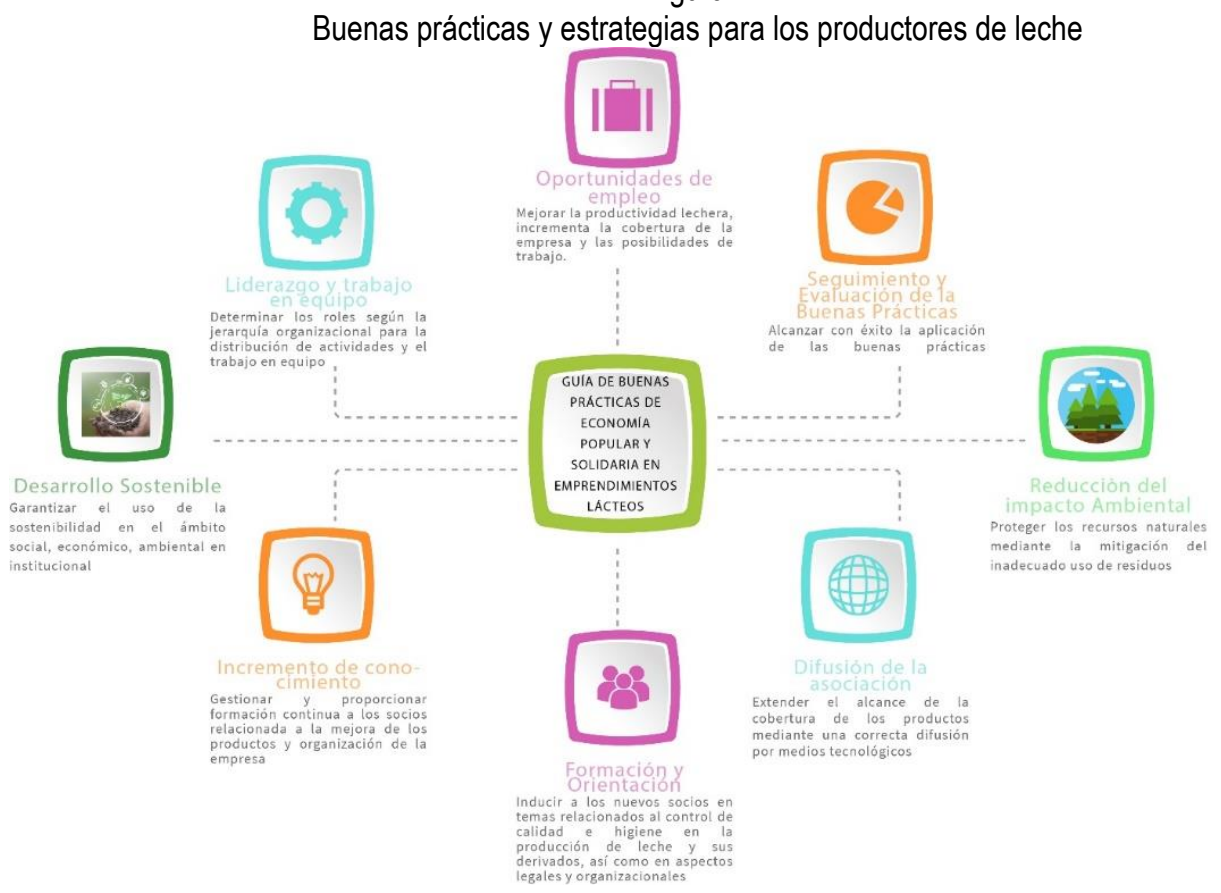

Fuente: Elaboración propia.

Tabla 1. Propuesta de buenas prácticas y estrategias para las empresas asociativas productoras de leche

\begin{tabular}{|c|c|}
\hline Buena Práctica & Estrategia \\
\hline $\begin{array}{l}\text { 1. Oportunidades de empleo: } \\
\text { Disminuir la tasa de desempleo y mejora la } \\
\text { calidad de vida de sus habitantes. Es } \\
\text { importante, que toda organización mejore su } \\
\text { productividad para que, como consecuencia } \\
\text { de esto incremente sus socios. (Derivada de } \\
\text { la falta de formación, falta de guías o modelo } \\
\text { de buenas prácticas, la visión del impacto en } \\
\text { las comunidades de las buenas prácticas) }\end{array}$ & $\begin{array}{l}\text { Dinamizar el aparato productivo lechero local, } \\
\text { mediante el impulso de las vocaciones } \\
\text { productivas. } \\
\text { Generar empleo digno en los socios y } \\
\text { trabajadores para transitar de un ingreso de } \\
\text { supervivencia a un ingreso que garantice el } \\
\text { Buen Vivir de cada uno de sus integrantes. }\end{array}$ \\
\hline $\begin{array}{l}\text { 2. Incremento de conocimiento: } \\
\text { Promover en los socios capacidades que los } \\
\text { lleven a estar preparados para identificar } \\
\text { oportunidades y tomar decisiones conjuntas. } \\
\text { (Derivada del desconocimiento acerca de la } \\
\text { ESS a la que pertenece la organización así } \\
\text { como de las buenas prácticas en este ramo). }\end{array}$ & $\begin{array}{l}\text { Gestionar y proporcionar formación continua a } \\
\text { los socios, relacionada con la mejora de los } \\
\text { productos y organización de la empresa, a } \\
\text { través de capacitaciones, talleres, seminarios, } \\
\text { entre otros, e incluso la autoeducación } \\
\text { socialmente responsable. }\end{array}$ \\
\hline
\end{tabular}


Buenas prácticas en emprendimientos lácteos, desde la economía social y solidaria en BibliánEcuador

\begin{tabular}{|c|c|}
\hline $\begin{array}{l}\text { 3. Liderazgo y trabajo en equipo: El } \\
\text { trabajo en equipo requiere de la participación } \\
\text { de las diferentes personas, en donde se } \\
\text { comparte habilidades y conocimientos, se } \\
\text { debe trabajar en espacios donde exista } \\
\text { confianza de expresarse y de cumplimiento de } \\
\text { las actividades, asumiendo el compromiso en } \\
\text { pro de alcanzar las metas y objetivos } \\
\text { propuestos. (Derivada de la falta de formación, } \\
\text { falta de guías o modelo de buenas prácticas. } \\
\text { Para fortalecer la visión acerca del impacto de } \\
\text { las buenas prácticas en la gestión) }\end{array}$ & $\begin{array}{l}\text { Determinar los roles de los actores sociales } \\
\text { según la jerarquía organizacional para la } \\
\text { distribución equitativa de actividades y el } \\
\text { trabajo en equipo. }\end{array}$ \\
\hline $\begin{array}{l}\text { 4. Formación y Orientación: Orientar y } \\
\text { otorgar los recursos necesarios a todos los } \\
\text { actores sociales para su familiarización con el } \\
\text { marco legal y su relación con el campo laboral, } \\
\text { de esta manera se garantiza un buen } \\
\text { desempeño dentro de la misma. (Derivada del } \\
\text { desconocimiento acerca de la ESS a la que } \\
\text { pertenece la organización así como de las } \\
\text { buenas prácticas en este ramo). }\end{array}$ & $\begin{array}{l}\text { Inducir a los nuevos socios en temas } \\
\text { relacionados al control de calidad e higiene en } \\
\text { la producción de leche y sus derivados, así } \\
\text { como en aspectos legales y organizacionales. }\end{array}$ \\
\hline $\begin{array}{l}\text { 5. Difusión de la asociación: Una } \\
\text { organización no crece ni se da conocer, si no } \\
\text { se cuenta con una adecuada difusión, es así } \\
\text { que, dentro de las buenas prácticas es } \\
\text { importante divulgar las acciones que se } \\
\text { realizan con la sociedad y desde la } \\
\text { solidaridad, pues hoy en día el uso de las } \\
\text { redes sociales y de la tecnología, se ha } \\
\text { convertido en una herramienta potente y } \\
\text { versátil. (Derivada del desconocimiento } \\
\text { acerca de la ESS a la que pertenece la } \\
\text { organización así como de las buenas prácticas } \\
\text { en este ramo, falta de formación). }\end{array}$ & $\begin{array}{l}\text { Extender el alcance de la cobertura de los } \\
\text { productos, mediante una correcta difusión por } \\
\text { medios tecnológicos desde la cooperación } \\
\text { solidaria. }\end{array}$ \\
\hline $\begin{array}{l}\text { 6. Reducción del Impacto Ambiental: } \\
\text { Instaurar el uso consiente de los recursos } \\
\text { naturales, usar eficientemente la energía y el } \\
\text { agua, disminuir la generación de residuos, } \\
\text { utilizar envases biodegradables, entre otros. } \\
\text { (Derivada del desconocimiento del desarrollo } \\
\text { de buenas prácticas de la ESS. Para fortalecer } \\
\text { la visión del desarrollo comunitario). }\end{array}$ & $\begin{array}{l}\text { Mitigación del inadecuado uso de residuos y } \\
\text { desechos sólidos. }\end{array}$ \\
\hline $\begin{array}{l}\text { 7. Desarrollo Sostenible: Coadyuvar al } \\
\text { gobierno nacional a implementar los Objetivos } \\
\text { de Desarrollo Sostenible (ODS), para } \\
\text { erradicar el hambre: "Poner fin al hambre, } \\
\text { lograr la seguridad alimentaria y la mejora de } \\
\text { la nutrición y promover la agricultura }\end{array}$ & $\begin{array}{l}\text { Invitar a los productores a hacer acuerdos de } \\
\text { precios justos de leche. } \\
\text { Crear espacios de relaciones directas entre } \\
\text { productor y consumidor final }\end{array}$ \\
\hline
\end{tabular}


sostenible" (Programa de las Naciones Unidad para el Desarrollo [PNUD], 2021). (Derivada del desconocimiento del desarrollo de buenas prácticas de la ESS. Para fortalecer la visión del desarrollo comunitario)

8. Seguimiento y Evaluación de la Buenas Prácticas: Dar seguimiento y evaluar las buenas prácticas, para medir su eficiencia, mejorando la eficacia y efectividad de la organización. (Derivada del desconocimiento del desarrollo de buenas prácticas de la ESS, de la debilidad en cuanto a los controles de calidad de los productos generados; debilidad en los procesos de higiene; escasa formación del personal)

Fuente: elaboración propia

A partir de las estrategias propuestas dentro de las buenas prácticas, se usó el modelo CANVAS, una herramienta que permite definir y crear modelos de negocios de manera simplificada. El modelo se visualiza en un lienzo, que presenta 4 grandes áreas: clientes, oferta, infraestructura y viabilidad económica, en un recuadro de 9 secciones que muestran los principales aspectos del negocio y su propuesta de valor.

CANVAS, permite crear, generar y entregar, ya sea productos o servicios, que satisfagan las necesidades de los clientes; esto a su vez asegura la viabilidad del organismo o empresa, desde la simplicidad de una estructura de negocios donde prevalezca la creación de valor (cuantitativo y ético) y el enfoque integral que facilite identificar los cambios, nuevas viabilidades y sus repercusiones sinérgicas con el entorno. En este caso particular se enfoca a las asociaciones de los productores de leche.

En el Cuadro 1, se determina el modelo para las organizaciones en función de las estrategias establecidas en la tabla 1:

1. Dinamizar el aparato productivo lechero local, mediante el impulso de las vocaciones productivas.

2. Generar empleo digno en los socios y trabajadores para transitar de un ingreso de supervivencia a un ingreso que garantice el Buen Vivir de cada uno de sus integrantes.

3. Gestionar y proporcionar formación continua a los socios, relacionada con la mejora de los productos y organización de la empresa, a través de capacitaciones, talleres, seminarios, entre otros, e incluso la autoeducación socialmente responsable.

4. Determinar los roles de los actores sociales según la jerarquía organizacional para la distribución equitativa de actividades y el trabajo en equipo.

5. Inducir a los nuevos socios en temas relacionados al control de calidad e higiene en la producción de leche y sus derivados, así como en aspectos legales y organizacionales. 
Buenas prácticas en emprendimientos lácteos, desde la economía social y solidaria en BibliánEcuador

6. Extender el alcance de la cobertura de los productos, mediante una correcta difusión por medios tecnológicos desde la cooperación solidaria.

7. Mitigación del inadecuado uso de residuos y desechos sólidos.

8. Invitar a los productores a hacer acuerdos de precios justos de leche.

9. Crear espacios de relaciones directas entre productor y consumidor final

10. Implementar programas de seguimiento y evaluación de las buenas prácticas social y solidaria en el ámbito lácteo.

\section{Cuadro 1}

Modelo de negocios para la producción lechera, basado en el Modelo CANVAS

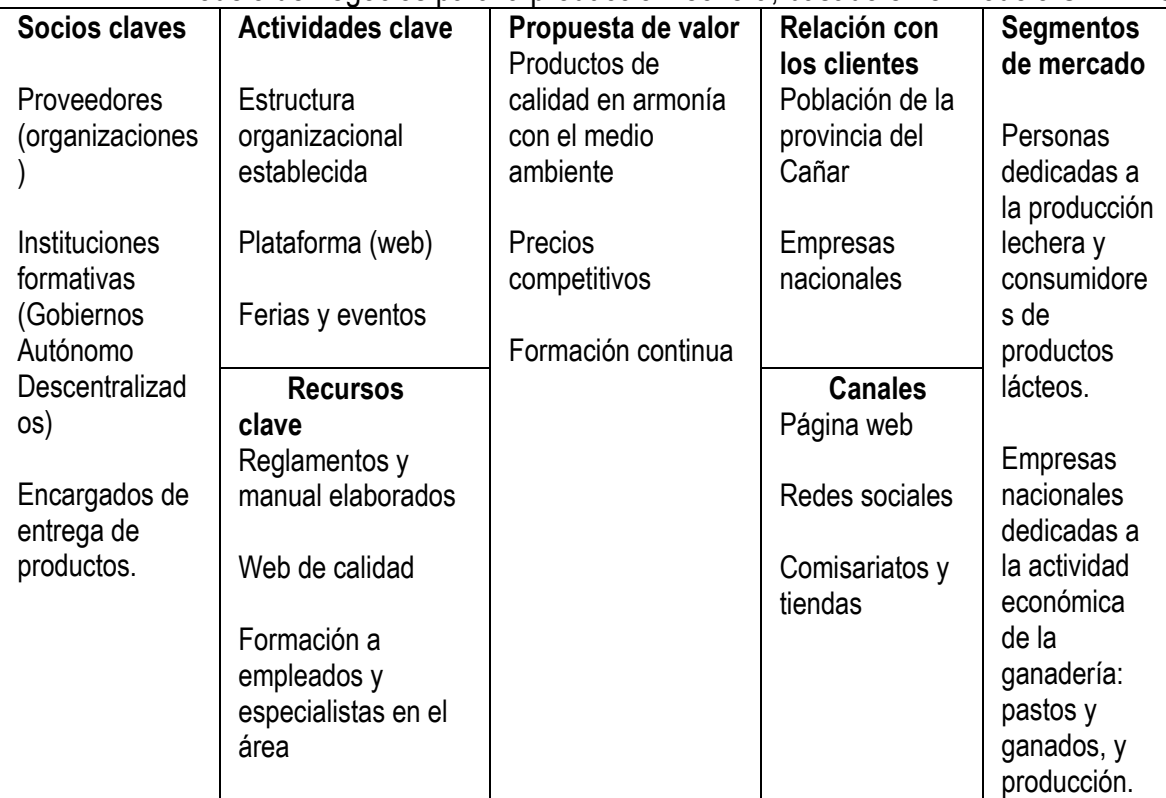

\section{Estructura de costes}

Publicidad en redes sociales

Elaboración, mantenimiento y actualización constante del sitio web

Compra de insumos básicos

Infraestructura tecnológica: compra de maquinaria y equipos

Sueldos y salarios del personal

Gastos de producción

\section{Fuentes de ingresos}

Venta directa de los productos

Patrocinio de marcas

Fuente: Elaboración propia. 


\section{Conclusiones}

Las asociaciones productoras de leche del cantón Biblián-Ecuador, deben dar muestras de su capacidad para armonizar la obtención de beneficios económicos y financieros, resultantes de la producción de leche con la preservación del bienestar de las personas (empleados, socios y clientes), la salud del rebaño (ganado) y el medio ambiente, todo ello a partir de la puesta en ejecución de buenas prácticas en el proceso productivo. Considerando, además, los principios que rigen a las organizaciones de la ESS.

Es clave que los asociados a estas organizaciones tomen conciencia de su rol en la economía del cantón y del país, ya que como productores son la base principal en la cadena de valor. En este aspecto, la explotación de leche debe tener la oportunidad de añadir valor a su producto incorporando métodos adecuados de producción que estén acordes a la satisfacción de los diferentes eslabones en la cadena de suministro.

El levantamiento de información en las asociaciones, puso en evidencia una serie de falencias en la gestión de los procesos administrativos, productivos, estructurales, entre otros, que afectan la productividad de la leche y sus derivados, lo que lleva a la urgente necesidad de revisar sistémicamente sus procesos, en aras de garantizas resultados positivos para ellas y la comunidad en la cual convergen.

En este contexto, se generaron estrategias dentro de la identificación de las buenas prácticas de economía social y solidaria con la finalidad de mejorar el uso de las mismas, así también, mediante estrategias se propone un modelo de negocios que permita potencializar la comercialización y mejorar la productividad de la leche y sus derivados.

Para futuras investigaciones se considera propicio analizar las unidades económicas sociales y solidarias de leche en el cantón con el objeto de elaborar una tipología de acuerdo a la realidad ecuatoriana en concordancia con las políticas públicas, que sea lo bastante flexible para que sirva de referente a otras actividades económicas.

\section{Referencias Bibliográficas}

Amagua Puga, Rubén; Coronel Feijó, Manuel; Quintana Mena, Tatiana; Reyes Merino, Carlos (2016). Guía de Buenas Prácticas de Manufactura para micro y pequeñas empresas Lácteas. Fortaleciendo la economía social y solidaria. http://sitp.pichincha.gob.ec/repositorio/diseno paginas/archivos/Gu\%C3\%ADa $\% 20 \mathrm{de} \%$ 20Buenas $\% 20 \mathrm{Pr} \% \mathrm{C} 3 \% \mathrm{~A} 1 \mathrm{cticas} \% 20 \mathrm{de} \% 20$ Manufactura $\% 20$ Micro\%20y $\% 20 \mathrm{Peque} \% \mathrm{C}$ 3\%B1as\%20Empresas \%20L\%C3\%A1cteas.pdf

Asamblea Constituyente (2008). Constitución de la República del Ecuador. Publicada en el Registro Oficial No. 44920 de octubre de 2008. https://www.asambleanacional.gob.ec/sites/defaultffiles/documents/old/constitucion_de bolsillo.pdf

Asamblea Nacional de la República del Ecuador (2011). Ley Orgánica de la Economía Popular y Solidaria y del Sector Financiero Popular y Solidario. Oficio N T.4887-SNJ-11-664. Quito, 28 de abril de 2011. República del Ecuador.

Ayala Zaldaña, Karla Patricia; Berltrán Flores, Isis Beatriz (2011). Economía Solidaria: Alternativa a la delincuencia y el desempleo. El caso de Santa Marta. Tesis pregrado. 
Buenas prácticas en emprendimientos lácteos, desde la economía social y solidaria en BibliánEcuador

Licenciado en Economía Universidad Centroamericas José Simeon Cañas. Antiguo Cuscatlan. El Salvador. http://www.socioeco.org/bdf_fiche-document-2826_es.html

Bretos, Ignacio; Díaz-Foncea, Millan; Marcuello, Chaime y Marcuello, Carmen. (2018). Cooperativas, capital social y emprendimiento: Una perspectiva teórica. REVESCO. Revista de Estudios Cooperativos, 128. España (Pp. 76-98). https://doi.org/10.5209/REVE.59775

Bonifaz García, Nancy y Requelme, Narcisa de Jesús. (2011). Buenas prácticas de ordeño y la calidad higiénica de la leche en el Ecuador. La Granja, 14 (2). Ecuador (Pp. 45-57). https://doi.org/10.17163/lgr.n14.2011.04

Castelao, María (2016). Las políticas públicas y su visión de la economía social y solidaria en Argentina. Revista Mexicana de Ciencias Políticas y Sociales. (227). México (Pp. 349378). https://doi.org/10.1016/S0185-1918(16)30032-0

Centro de la Industria Láctea del Ecuador (S/F). Industria láctea apoya desarrollo social y económico a escala nacional. https://www.cilecuador.org/post/industria-l\%C3\%A1cteaapoya-desarrollo-social-y-econ\%C3\%B3mico-a-escala-nacional

Centro de la Industria Láctea del Ecuador (2021). Industria láctea ecuatoriana: trabajo y compromiso por fortalecer la economía nacional.

https://www.cilecuador.org/post/industria-1\%C3\%A1ctea-ecuatoriana-trabajo-ycompromiso-por-fortalecer-la-econom\%C3\%ADa-nacional

Chong-González, Elizabeth Guadalupe (2017). El emprendimiento como agente potenciador del desarrollo económico local en el espacio rural. Revista de Desarrollo Económico. 4 (11).

México

(Pp. 26-36). https://www.ecorfan.org/bolivia/researchjournals/Desarrollo_Economico/vol4num11/Rev ista de Desarrollo_Econ\%C3\%B3mico_V4_N11_3.pdf

Da Ros, Giuseppina (2001). Realidad y Desafíos de la Economía Solidaria Iniciativas comunitarias y cooperativas en el Ecuador. Editorial ABYA-YALA. Ecuador.

Economistas sin Fronteras. (2017). Informe de Evaluación y Buenas prácticas. Proyecto Yo hago prácticas en la Economía Social y Solidaria. Movimiento por la Paz, Economistas sin Fronteras, Obra Social "la Caixa". Extraído de http://ecosfron.org/wpcontent/uploads/Informe-evaluaci\%C3\%B3n-y-buenas-pr\%C3\%A1cticas-proyecto.pdf

Fajardo, Gemma (2009). La economía social en las leyes. CIRIEC-España, Revista de Economía Pública, Social y Cooperativa, (66), España. (Pp. 5-35). http://base.socioeco.org/docs/fajardo. la es_en las leyes.pdf

Fajardo, Gemma. (2018). La identificación de las empresas de economía social en España. Problemática jurídica. REVESCO. Revista de Estudios Cooperativos. 128. España (99-126). https://doi.org/10.5209/REVE.60209

Fernández, Gabriela y Falconi, David. (2012). Cinco años de política económica para el buen vivir. Revista Ecuador Económico, (05). Ecuador (Pp.12-18). https://issuu.com/economiaecuador/docs/ec-5-web

Fonteneau, Bénedicte; Neamtan, Nancy; Wanyama, Federick; Pereira Morais, Leandro y De Poorter, Mathieu (2010). Documento de trabajo: Economía social y solidaria: construyendo un entendimiento común. (primera edición) Centro Internacional de Formación de la Organización Internacional del Trabajo 2010. Italia. 
https://www.ilo.org/wcmsp5/groups/public/---ed_emp/---emp_ent/--coop/documents/publication/wcms 546400.pdf

Gibson, Katherine y Graham, Julie (2011). Una política poscapitalista. Siglo del Hombre Editores-Editorial Pontificia Universidad Javeriana. Colombia.

González Ibarra, Miguel Rodrigo (2020). Emprendimiento social para el desarrollo comunitario en México: el caso del centro de desarrollo comunitario sustentable (CEDECOSU) del CREFAL en Pátzcuaro, Michoacán. Revista Exitus. 10. México (Pp. 01-28). https://doi.org/10.24065/2237-9460.2020v10n1ID1465

Gómez Calvo, Verónica (2013). Acercamiento a las prácticas de la economía social, la economía solidaria y la economía del bien común, ¿qué nos ofrecen? Revista Castellano-Manchega de Ciencias Sociales, 15. España (pp. 111-124). https://doi.org/10.20932/barataria.v0i15.86

Guerra, Pablo (2012). Miradas globales para otra economía. SETEM, España. http://www.relats.org/documentos/ESSPublicaciones4Guerra.pdf

Instituto Nacional de Economía Popular y Solidaria (2014). IEPS impulsa el saber-hacer para capacitar a los actores del Programa Hilando el Desarrollo. https://www.economiasolidaria.gob.ec/ieps-impulsa-el-saber-hacer-para-capacitar-alos-actores-del-programa-hilando-el-desarrollo/

Lácteos Latam (2020). Industria láctea clave para la reactivación económica del Ecuador. https://www.lacteoslatam.com/sectores/36-leches/4064-industria-1\%C3\%A1ctea-clavepara-reactivaci\%C3\%B3n-econ\%C3\%B3mica-en-ecuador.html

Ministerio de Salud Pública (2014). Reglamento de etiquetado de alimentos procesados para consumo humano. Acuerdo 5103. http://extwprlegs1.fao.org/docs/pdf/ecu175772.pdf

Mogrovejo, Rodrigo; Mora, Alberto y Vanhuynegem, Philippe (2012).El cooperativismo en América Latina. Una diversidad de contribuciones al desarrollo sostenible. Oficina de la OIT para los Países Andinos. https://www.ilo.org/wcmsp5/groups/public/--americas/---ro-lima/documents/publication/wcms 188087.pdf

Ojeda, Sara. (2009). La economía solidaria, un modelo de desarrollo social caso de estudio en el Ecuador. Trabajo de Grado de la Facultad de Economía. Pontificia Universidad Católica del Ecuador. http://repositorio.puce.edu.ec/handle/22000/5281

Organización de las Naciones Unidas para la Alimentación y la Agricultura [FAO] y la Federación Internacional de la Leche [FIL] (2012). Guía de buenas prácticas en explotaciones lecheras. Directrices FAO: Producción y Sanidad Animal. http://www.fao.org/3/ba0027s/ba0027s00.pdf

Orrego, Clara y Arboleda, Olga (2006). Las organizaciones de economía solidaria: Un modelo de gestión innovador. http://www.redalyc.org/pdf/2250/225020897004.pdf

Pérez Paredes, Alfredo; Torres-Flórez, Dagoberto; Torralba Flores, Amado; Salgado Cifuentes y Wilson, Fernando (2020). Características y elementos del fenómeno emprendedor en Villavicencio, Colombia. Cuadernos Latinoamericanos de Administración, 16 (30). Colombia (Pp.1-10). https://www.redalyc.org/journal/4096/409663283005/409663283005.pdf 
Buenas prácticas en emprendimientos lácteos, desde la economía social y solidaria en BibliánEcuador

Presidencia de la República (2002). Reglamento de Buenas Prácticas para Alimentos Procesados. Decreto Ejecutivo 3253, Registro Oficial 696. Material MIPRO. http://www.epmrq.gob.ec/images/lotaip/leyes/rbpm.pdf

Programa de la Naciones Unidas para el Desarrollo [PNUD], (2021). Objetivos de Desarrollo Sostenible. $\quad$ https://www.ec.undp.org/content/ecuador/es/home/sustainabledevelopment-goals.html

Razeto, Luis (1999). La economía solidaria: concepto, realidad y proyecto. https://www.luisrazeto.net/content/la-econom\%25C3\%25ADa-solidaria-conceptorealidad-y-proyecto

Red de Redes de Economía Alternativa y Solidaria. (2011). Carta de Principios de la Economía Solidaria. REAS. https://www.economiasolidaria.org/carta-de-principios-de-laeconomia-solidaria/

Red Intercontinental de Promoción de la Economía Social y Solidaria (2015). RIPESS. Red Intercontinental de Promoción de la Economía Social y Solidaria. http://www.ripess.org/quienes-somos/sobre-ripess/?lang=es

Rodríguez, Axel y Sánchez, Edgar (2016). Análisis comparativo entre empresas de emprendimiento social y tradicionales por medio de indicadores financieros clave. $\begin{array}{lllll}\text { Vinculategica. } & 2 & \text { (1). } & \text { México } & \text { (pp. 2310-2338). }\end{array}$ http://www.web.facpya.uanl.mx/vinculategica/Revistas/R2/2310\%20-\%202338\%20-

\%20ANILISIS\%20COMPARATIVO\%20ENTRE\%20EMPRESAS\%20DE\%20EMPREND IMIENTO\%20SOCIAL\%20Y\%20TRADICIONALES\%20POR\%20MEDIO\%20DE\%20IN DICADORES\%20FINANCIEROS\%20CLAVE.pdf

Ruiz Revelo, Emilia Jael (2015). La Economía Social y Solidaria como modelo de desarrollo emergente en el Ecuador. Disponible en: http://repositorio.puce.edu.ec/bitstream/handle/22000/10538/La\%20econom\%C3\%ADa \%20social\%20y\%20solidaria\%20como\%20modelo\%20de\%20desarrollo\%20emergente $\% 20$ en $\% 20$ el $\% 20$ Ecuador\%20-\%20Emilia\%20Ruiz.pdf?sequence=1

Sarria Icaza, Ana Mercedes (2002). Economía solidaria, prácticas cooperativas y desarrollo local en el sur de Brasil. CIRIEC-España, Revista de Economía Pública, Social y Cooperativa, (43), $\quad$ España. $\quad$ (Pp. https://base.socioeco.org/docs/_pdf_174_17404304.pdf

Singer, Paúl (2002). Introducción a la economía solidaria. Editorial Fundación Perseu Abramo. Brasil

Subijana, Telémaco y Russo, Vicente (2011). Los Estados deben marcar el rumbo económico, nuestras economías no pueden quedar libradas al juego del mercado global. Entrevista al reconocido economista José Coraggio. http://www.coraggioeconomia.org/ilc/archivos\%20para\%20descargar/Los_Estados_deb en marcar_el rumbo economico.pdf

Superintendencia de la Economía Popular y Solidaria (2021). Boletín sectorial de la economía popular y solidaria. Cooperativas, Asociaciones y Organizaciones comunitarias. Evolución de la economía popular y solidaria. https://app.powerbi.com/view?r=eyJrljoiYWM2MmQ2NDktOTI5Yi00NDUzLWE3ODMtY 
zc1N2E00DQ5ZmQ4liwidCI6ImMwNWUxMWU1LTcwNmMtNGNIZi1iYTVILTE4ZWVk YmYxMDAzNyJ9

Tello, Mario (2006). Las teorías del desarrollo económico local y la teoría y práctica del proceso de descentralización en los países en desarrollo. https://departamento.pucp.edu.pe/economia/documento/las-teorias-del-desarrolloeconomico-local-y-la-teoria-y-practica-del-proceso-de-descentralizacion-en-los-paisesen-desarrollo/

Villoch, Alejandra M. y Ponce, P. (2010). Buena práctica de producción lechera para Cuba. Estrategia para su aplicación. Rev. Salud Anim. 32(3). Cuba (Pp. 192-197). http://scielo.sld.cu/scielo.php?script=sci_arttext\&pid=S0253570X2010000300009\&lng=es\&nrm=iso

Zhunio, Galo (2021). La Economía Popular y Solidaria: Un Modelo para el Desarrollo. Tesis de Maestría en Finanzas y Economía Empresarial. Universidad Católica Santiago de Guayaquil. Ecuador. http://repositorio.ucsg.edu.ec/bitstream/3317/15873/1/T-UCSGPOS-MFEE-237.pdf 